\title{
Commentary on "Measurement and Correlation of the Solubility of Telmisartan (Form A) in Nine Different Solvents from 277.85 to 338.35 K"
}

\author{
William E. Acree Jr. ${ }^{1}$ - Melissa Y. Horton ${ }^{1} \cdot$ Elizabeth Higgins $^{1}$ • \\ Michael H. Abraham ${ }^{2}$
}

Published online: 11 November 2016

(C) Springer Science+Business Media New York 2016

\begin{abstract}
Several errors and shortcomings in the paper by Liu et al. (J. Solution Chem. 45:932-946, 2016) concerning the calculation of Abraham model solution descriptors of telmisartan are discussed. The authors' calculated solute descriptors give an unreasonable value for the water to 1-octanol partition coefficient of telmisartan, which is eight orders of magnitude smaller than values predicted by other methods. The correct computation methodology for determining solute descriptors from experimental solubility data is presented.
\end{abstract}

Keywords Abraham model solute descriptors · Solubility of telmisartan · Organic solvents · Partition coefficients

In a recent paper appearing in This Journal [1] Liu et al. reported the solubility of telmisartan (form A) in nine different organic solvents in the temperature range from 277.88 to $338.35 \mathrm{~K}$. The solubilities were measured using a laser monitoring method that detected the temperature at which the last added portion of solute did not dissolve. The authors described the temperature dependence of the measured mole fraction solubilities, $x_{1}$, in each individual organic solvent in terms of both the Apelblat equation:

$$
\ln x_{1}=A_{A}+B_{A} / T+C_{A} \ln (T)
$$

and $\lambda h$ equation:

William E. Acree Jr.

acree@unt.edu

1 Department of Chemistry, University of North Texas, 1155 Union Circle Drive \#305070, Denton, TX 76203, USA

2 Department of Chemistry, University College London, 20 Gordon Street, London WC1H OAJ, UK 


$$
x_{1}=\frac{1}{(1 / \lambda)\left\{\exp \left[\lambda h\left(\frac{1}{T}-\frac{1}{T_{\mathrm{m}}}\right)\right]-1\right\}+1}
$$

where $T$ and $T_{\mathrm{m}}$ correspond to the system temperature and melting point temperature of telmisartan, respectively. The empirical model curve-fit constants are denoted as $A_{\mathrm{A}}, B_{\mathrm{A}}$, $C_{\mathrm{A}}, \lambda$ and $h$ in Eqs. 1 and 2.

As part of the study the authors used the Abraham model:

$$
\log _{10} P=c_{\mathrm{p}}+e_{\mathrm{p}} \cdot E+s_{\mathrm{p}} \cdot S+a_{\mathrm{p}} \cdot A+b_{\mathrm{p}} \cdot B+v_{\mathrm{p}} \cdot V
$$

to calculate the solute's excess molar refraction descriptor $(E)$, dipolarity/polarizability descriptor $(S)$, hydrogen-bond acidity and basicity descriptors ( $A$ and $B)$, and McGowan characteristic volume $(V)$. The calculated numerical values, $E=0.71 ; S=1.35$; $A=-0.16 ; B=1.33$; and $V=1.27$, were obtained by fitting the data about $298 \mathrm{~K}$. The Abraham model, Eq. 3, correlates the logarithm of the water-to-solvent partition coefficient, $\log _{10} P$, which can be estimated as the logarithm of the solute's molar solubility in the organic solvent divided by the solute's aqueous molar solubility. No reference was given in the paper [1] for the source of the aqueous molar solubility, and the authors did not state how the $\log _{10} P$ values were obtained.

The purpose of the present communication is to point out several errors in the authors' curve-fitting results and several shortcomings in the solute descriptor computations. First, the Apelblat model equation coefficients that Liu et al. report in Table 4 of their paper are not consistent with the Apelblat model. If one simply substitutes the numerical values from Table 4 into Eq. 1 above one obtains $10^{3} x_{1}$, rather than $x_{1}$. The back-calculated mole fraction solubilities differ from the experimental values by a factor of 1000 . Readers should be aware of this problem when using the tabulated equation coefficients to calculate mole fraction solubilities at other temperatures.

Second, the calculation methodology for obtaining the Abraham model solute descriptors is seriously flawed. The McGowan characteristic volume should not be treated as a curve-fit parameter, but rather calculated from the molecular structure, atomic sizes and number of bonds as described elsewhere [2]. Correctly calculated, the McGowan volume for telmisartan is $V=3.9785$. The $E$ solute descriptor can be estimated by the PharmaAlgorithm software [3], which is based on molecular structure considerations using fragment group values $[4,5]$, or calculated using a measured value (liquid solute) or an estimated value (solid solute) for the solute's refractive index. The refractive index of solid solutes can be estimated using the (free) ACD software [6]. The value of $E$ that we calculate is $E=4.61$. This leaves us with three solute descriptors $(S, A$ and $B)$ to be calculated from the measured experimental solubility data. In the present case, the aqueous molar solubility of telmisartan is unknown, which leaves us with a fourth value to be calculated.

Liu et al. measured the solubility of telmisartan in nine different organic solvents; however, not all of the experimental data is useable in terms of calculating the Abraham descriptors since the solute is capable of dimerization. Carboxylic acids are known to dimerize in alkane and aromatic hydrocarbon solutes, and in other nonpolar solvents. The numerical values of the solute descriptors of monomeric carboxylic acids are different than the numerical values of dimeric carboxylic acids. One must select solubility data for solvents in which telmisartan is expected to exist almost entirely in monomeric form. Solubility data in dichloromethane, trichloromethane, benzene and toluene is eliminated from consideration because of dimerization concerns. This leaves us with solubility data in 
methanol, ethanol, 2-propanol, propanone and ethyl acetate for use in the solute descriptor calculations. We have three solute descriptors and an unknown aqueous solubility to be calculated from five experimental $\log _{10} P$ equations - one equation for each of the five different solvents in which telmisartan exists predominantly in monomeric form.

It is possible to double the number of equations by converting all the $\log _{10} P$ values into $\log _{10} K$ values through Eq. 4, where $K_{\mathrm{w}}$ is the gas-to-water partition coefficient (unit-less if concentrations in the gas phase and the aqueous phase are both in $\mathrm{mol} \cdot \mathrm{L}^{-1}$ )

$$
\begin{gathered}
\log _{10} P=\log _{10} K-\log _{10} K_{\mathrm{w}} \\
\log _{10} K=c_{\mathrm{k}}+e_{\mathrm{k}} \cdot E+s_{\mathrm{k}} \cdot S+a_{\mathrm{k}} \cdot A+b_{\mathrm{k}} \cdot B+l_{\mathrm{k}} \cdot L
\end{gathered}
$$

Then if, say, five $\log _{10} P$ values are available, five more equations in $\log _{10} K$ can be used, and two more equations in $\log _{10} K_{\mathrm{W}}$ are also available

$$
\begin{aligned}
& \log _{10} K_{\mathrm{w}}=-0.994+0.577 \cdot E+2.549 \cdot S+3.813 \cdot A+4.841 \cdot B-0.869 \cdot V \\
& \log _{10} K_{\mathrm{w}}=-1.271+0.822 \cdot E+2.743 \cdot S+3.904 \cdot A+4.814 \cdot B-0.213 \cdot L
\end{aligned}
$$

$\log _{10} K_{\mathrm{w}}$ will normally have to be found by trial-and-error, so that two extra descriptors ( $L$ and $\log _{10} K_{\mathrm{w}}$ ) have to be determined. However, starting with five $\log _{10} P$ values, no less than ten equations will be available to calculate the four missing descriptors $(S, A, B$, and $L$ ), the aqueous molar solubility of telmisartan $\left(C_{\mathrm{w}}\right)$ and $\log _{10} K_{\mathrm{w}}$. This method has been applied extensively to the solubility of solids by Acree et al. [7-10].

We have compiled in Table 1 the numerical values of the $\log _{10} P$ and $\log _{10} K$ equation coefficients for methanol, ethanol, 2-propanol, acetone and ethyl acetate. Preliminary regression analysis showed that the methanol data were outliers. The two data points were removed from the analysis. The ten remaining equations were solved simultaneously using Microsoft Solver software to yield numerical values of: $E=4.610 ; S=3.651 ; A=0.565$; $B=2.130 ; V=3.9785 ; L=23.155 ; \log _{10} C_{\mathrm{w}}=-9.436$; and $\log _{10} K_{\mathrm{w}}=20.021$ with the overall standard error being $\mathrm{SE}=0.048 \log _{10}$ units. As an informational note, the calculated

Table $1 \log _{10} P$ and $\log _{10} K$ equation coefficients for the solvents considered in the present commentary

\begin{tabular}{lrrrrrrr}
\hline Partition system & \multicolumn{1}{c}{$c$} & \multicolumn{1}{l}{$s$} & \multicolumn{1}{l}{$b$} & \multicolumn{1}{l}{$l$} & $v$ \\
\hline Gas-water/298 & -0.994 & 0.577 & 2.549 & 3.813 & 4.841 & 0.000 & -0.869 \\
Water-methanol & 0.276 & 0.334 & -0.714 & 0.243 & -3.320 & 0.000 & 3.549 \\
Water-ethanol & 0.222 & 0.471 & -1.035 & 0.326 & -3.596 & 0.000 & 3.857 \\
Water-2-propanol & 0.099 & 0.344 & -1.049 & 0.406 & -3.827 & 0.000 & 4.033 \\
Water-ethyl acetate & 0.328 & 0.369 & -0.446 & -0.700 & -4.904 & 0.000 & 4.150 \\
Water-propanone & 0.313 & 0.312 & -0.121 & -0.608 & -4.753 & 0.000 & 3.942 \\
Water-wet octanol & 0.088 & 0.562 & -1.054 & 0.034 & -3.460 & 0.000 & 3.814 \\
Gas-water/298 & -1.271 & 0.822 & 2.743 & 3.904 & 4.814 & -0.213 & 0.000 \\
Gas-methanol & -0.039 & -0.338 & 1.317 & 3.826 & 1.396 & 0.773 & 0.000 \\
Gas-ethanol & 0.017 & -0.232 & 0.867 & 3.894 & 1.192 & 0.846 & 0.000 \\
Gas-2-propanol & -0.048 & -0.324 & 0.713 & 4.036 & 1.055 & 0.884 & 0.000 \\
Gas-ethyl acetate & 0.182 & -0.352 & 1.316 & 2.891 & 0.000 & 0.916 & 0.000 \\
Gas-propanone & 0.127 & -0.387 & 1.733 & 3.060 & 0.000 & 0.866 & 0.000 \\
\hline
\end{tabular}


Absolv descriptors are [11]: $E=4.61, S=3.56, A=0.57, B=1.59, V=3.9785$, and $L=21.58$ in reasonably good agreement with the values that we have calculated.

The solute descriptors that were reported by Liu et al. are unrealistic and are not consistent with the molecular structure of telmisartan. A very simple way of checking any set of descriptors is to calculate $\log _{10} P$ for the water-wet octanol system by combining the descriptor set with the equation for water-wet octanol partition coefficients (Table 1). The descriptors of Liu et al. yield a value of -0.70 , our descriptors yield 6.64, and calculated values of $\log _{10} P$ are 7.29 (BioLoom), [12] 7.73 (ACD), [6, 13] 6.04 (ChemAxon), [13] 6.95 (Absolv) [11] and 8.42 (EPI). [14] Our value is within the range of the various calculated values, whilst that calculated from the coefficients of Liu et al. [1] differs by some $8 \log$ units.

We urge workers who are interested in our method to study a number of published reviews [15-18]. That of Clarke and Mallon [18] is particularly detailed.

Acknowledgements Melissa Horton and Elizabeth Higgins thank the University of North Texas's Texas Academy of Math and Science (TAMS) program for a summer research award.

\section{References}

1. Liu, W., Guo, Y., Chen, J., Yu, X.: Measurement and correlation of the solubility of telmisartan (form A) in nine different solvents from 277.85 to 338.38 K. J. Solution Chem. 45, 932-946 (2016)

2. Abraham, M.H., McGowan, J.C.: The use of characteristic volumes to measure cavity terms in reversed phase liquid chromatography. Chromatographia 23, 243-246 (1987)

3. PharmaAlgorithms, ADME Boxes, Version 3.0, PharmaAlgorithms Inc., Toronto

4. Platts, J.A., Butina, D., Abraham, M.H., Hersey, A.: Estimation of molecular linear free energy relation descriptors using a group contribution approach. J. Chem. Inf. Comput. Sci. 39, 835-845 (1999)

5. Platts, J.A., Abraham, M.H., Butina, D., Hersey, A.: Estimation of molecular linear free energy relationship descriptors by a group contribution approach. 2. Prediction of partition coefficients. J. Chem. Inf. Comput. Sci. 40, 71-80 (2000)

6. Advanced Chemistry Development, Toronto. The ACD Freeware can be accessed at http://www. acdlabs.com/

7. Abraham, M.H., Green, C.E., Acree Jr., W.E., Hernandez, C.E., Roy, L.E.: Descriptors for solutes from the solubility of solids: trans-stilbene as an example. J. Chem. Soc. Perkin Trans. 2, 2677-2682 (1998)

8. Hoover, K.R., Coaxum, R., Pustejovsky, E., Acree Jr., W.E., Abraham, M.H.: Thermochemical behavior of dissolved carboxylic acid solutes: part 5-mathematical correlation of 3,5-dinitrobenzoic acid solubilities with the Abraham solvation parameter model. Phys. Chem. Liq. 42, 457-466 (2004)

9. Abraham, M.H., Smith, R.E., Luchtefeld, R., Boorem, A.J., Luo, R., Acree Jr., W.E.: Prediction of solubility of drugs and other compounds in organic solvents. J. Pharm. Sci. 99, 1500-1515 (2010)

10. Abraham, M.H., Acree Jr., W.E., Brumfield, M., Hart, E., Pipersburgh, L., Mateja, K., Dai, C., Grover, D., Zhang, S.: Deduction of physicochemical properties from solubilities: 2,4-dihydroxybenzophenone, biotin, and caprolactam as examples. J. Chem. Eng. Data 60, 1440-1446 (2015)

11. Absolv, version 5.0; Advanced Chemistry Development: Toronto

12. BioLoom, BioByte Corp, Claremont

13. ChemSpider, www.chemspider.com/

14. EPI Suite TM, United States Environmental Protection Agency, 2000-2012

15. Abraham, M.H.: Scales of hydrogen bonding: their construction and application to physicochemical and biochemical processes. Chem. Soc. Rev. 22, 73-83 (1993)

16. Abraham, M.H., Ibrahim, A., Zissimos, A.M.: The determination of sets of solute descriptors from chromatographic measurements. J. Chromatogr. A 1037, 29-47 (2004)

17. Poole, C.F., Ariyasena, T.C., Lenca, N.: Estimation of the environmental properties of compounds from chromatographic properties and the solvation parameter method. J. Chromatogr. A 1317, 85-104 (2013)

18. Clarke, E.D., Mallon, L.: The determination of Abraham descriptors and their application to crop protection research. In: Jeschke, P., Krämer, W., Schirmer, U., Witschel, M. (eds.) Modern Methods in Crop Protection Research. Wiley, Weinheim (2012) 